\title{
Correction to: Remote Monitoring and Predictive Maintenance of Medical Devices
}

Mohammed Saleh Ahmed Qaid, Anas Mohd Noor, Ahmad Nasrul Norali, Zulkarnay Zakaria, A. Z. Ahmad Firdaus, Asyraf Hakimi Abu Bakar, and Chong Yen Fook

\section{Correction to:}

Chapter "Remote Monitoring and Predictive Maintenance of Medical Devices" in: F. Thakkar et al. (eds.), Proceedings of the International e-Conference on Intelligent Systems and Signal Processing, Advances in Intelligent Systems and Computing 1370, https://doi.org/10.1007/978-981-16-2123-9_56

In the original version of the book, the author name has been updated from "Mohammed Salleh Ahmed Qaid" to "Mohammed Saleh Ahmed Qaid" in the Chapter "Remote Monitoring and Predictive Maintenance on Medical Devices". The chapter and book have been updated with the changes. 